\section{RSP}

http://www.rsp.fsp.usp.br/
Revista de Saúde Pública

\title{
Predição de absenteísmo docente na rede pública com machine learning
}

\author{
Fernando Timoteo Fernandes ${ }^{1, \prime \prime}$ iD, Alexandre Dias Porto Chiavegatto Filho ${ }^{\text {III }}$ \\ I Universidade de São Paulo. Faculdade de Saúde Pública. Programa de Pós-Graduação em Saúde Pública. São \\ Paulo, SP, Brasil \\ "Fundacentro. São Paulo, SP, Brasil \\ III Universidade de São Paulo. Faculdade de Saúde Pública. São Paulo, SP, Brasil
}

\section{RESUMO}

OBJETIVO: Predizer o risco de ausência laboral decorrente de morbidades dos docentes que atuam na educação infantil na rede pública municipal, com o uso de algoritmos de machine learning.

MÉTODOS: Trata-se de um estudo transversal utilizando dados secundários, públicos e anônimos da Relação Anual de Informações Sociais, selecionando professores da educação infantil que atuaram na rede pública municipal do estado de São Paulo entre 2014 e 2018 $(\mathrm{n}=174.294)$. Foram também vinculados dados da média de alunos por turma e número de habitantes no município. Os dados foram separados em treinamento e teste, utilizando os registros de 2014 a $2016(\mathrm{n}=103.357)$ para treinar cinco modelos preditivos e os dados de 2017 a $2018(n=70.937)$ para testar seus desempenhos em dados novos. A performance preditiva dos algoritmos foi avaliada por meio do valor da área abaixo da curva ROC (AUROC).

RESULTADOS: Todos os cinco algoritmos testados apresentaram área abaixo da curva acima de 0,76 . $\mathrm{O}$ algoritmo com melhor performance preditiva (redes neurais artificiais) obteve 0,79 de área abaixo da curva, com acurácia de 71,52\%, sensibilidade de 72,86\%, especificidade de 70,52\% e kappa de 0,427 nos dados de teste.

Correspondência:

Fernando Timoteo Fernandes

Rua Capote Valente, 710

05409-002 São Paulo, SP, Brasil

E-mail: fernando.fernandes@

fundacentro.gov.br

Recebido: 18 mai 2020

Aprovado: 17 ago 2020

Como citar: Fernandes FT,

Chiavegatto Filho ADP. Predição

de absenteísmo docente na rede

pública com machine learning. Rev

Saude Publica. 2021;55:23.

https://doi.org/10.11606/s1518-

8787.2021055002677

Copyright: Este é um artigo de acesso aberto distribuído sob os termos da Licença de Atribuição Creative Commons, que permite uso irrestrito, distribuiç̧ão e reprodução em qualquer meio, desde que o autor e a fonte originais sejam creditados.

CONCLUSÃO: É possível predizer casos de afastamentos por morbidade em docentes da rede pública com machine learning usando dados públicos. O melhor algoritmo apresentou melhor resultado da área abaixo da curva quando comparado ao modelo de referência (regressão logística). Os algoritmos podem contribuir para predições mais assertivas na área da saúde pública e da saúde do trabalhador, permitindo acompanhar e ajudar a prevenir afastamentos por morbidade desses trabalhadores.

DESCRITORES: Absenteísmo. Fatores de Risco. Aprendizado de Máquina Supervisionado. Professores Escolares. Educação Infantil. 


\section{INTRODUÇÃO}

Profissionais da área da educação básica apresentam altos índices de afastamento por doença relacionada ao trabalho, situando-se entre as primeiras posições no Brasil ${ }^{1}$. Entre as suas principais doenças, estão os distúrbios de voz, doenças do aparelho respiratório e do sistema osteomuscular, além de transtornos mentais e comportamentais ${ }^{2,3}$. Houve um crescimento recente de notificações relacionadas à saúde mental, como no caso do estado de São Paulo ${ }^{4}$. As condições de trabalho a que esses profissionais estão expostos, com longas jornadas, turmas numerosas ${ }^{5}$ e falta de reconhecimento, acabam agravando o problema, com consequências diretas na vida particular dos docentes ${ }^{6}$.

No entanto, ainda há poucos indicadores que analisam as condições dos docentes em cada etapa do ensino básico, desde a educação infantil até o ensino médio, áreas que apresentam distinções e especificidades no contexto de trabalho ${ }^{7}$. Estudos que buscam gerar indicadores normalmente utilizam valores agregados das diferentes etapas do ensino básico ${ }^{1}$ ou são baseados em inquéritos em regiões específicas que dependem da ação do respondente, podendo gerar altos custos operacionais ${ }^{8.9}$.

O uso de indicadores agregados para a análise de doenças pode omitir situações críticas de morbidade e não corresponder à realidade das diferentes condições de trabalho às quais os docentes estão expostos. O problema se agrava ao analisar professores da rede pública municipal, cujos dados de afastamento somente são acessíveis por meio de secretarias de saúde ou departamentos de perícias médicas de cada municípioº ${ }^{2}$ Uma forma de analisar a situação de morbidade por etapa de ensino e a relação do trabalho com o adoecimento de professores é buscar fontes de dados oficiais públicas, que possuam registros individualizados e atualizados com ampla cobertura geográfica.

Nesse cenário, a partir de 2011, o acesso aos dados provenientes do governo federal foi ampliado, mediante a publicação da Lei no 12.527 de 2011, conhecida como Lei de Acesso à Informação (LAI). Essa legislação permite que sejam solicitadas informações a qualquer órgão público e prevê que as entidades e órgãos públicos se antecipem e publiquem seus dados e informações na internet em formatos abertos e não proprietários, conhecidos como dados abertos ${ }^{10}$. Um exemplo é a Relação Anual de Informações Sociais (RAIS), instituída pelo Decreto $\mathrm{n}^{\circ}$ 76.900, de 23 de dezembro de 1975, atualmente sob responsabilidade do Ministério da Economia. Os dados são divulgados anualmente de forma pública e anônima, contendo registros individualizados com cobertura em todo o território nacional ${ }^{11}$.

A RAIS tem como principal finalidade subsidiar a geração de estatísticas do trabalho e do mercado formal (privado e estatutário) para as entidades governamentais. No entanto, também são disponibilizados os tipos de afastamento, como doença ou doença relacionada ao trabalho, sem informações da doença específica. O preenchimento é obrigatório a todas as empresas públicas e privadas desde $1977^{12}$. Nos dados de afastamento de professores na RAIS, verifica-se que em média mais de $40 \%$ dos professores da educação infantil se afastaram do trabalho ao menos uma vez ao ano devido a morbidades no período entre 2014 e 2018, superando as demais etapas do ensino básico e apontando para a necessidade de analisar esse grupo específico.

O presente estudo propõe construir modelos preditivos capazes de estimar o risco de ausência laboral dos docentes da educação infantil que atuam na rede pública municipal, considerando todos os municípios do estado de São Paulo, utilizando inteligência artificial e algoritmos de aprendizagem supervisionada (machine learning) conhecidos pelo alto desempenho, principalmente na área da saúde ${ }^{13}$. Espera-se que o melhor modelo proposto possa ser aplicado para estimar o número de afastamentos por doença em professores, utilizando dados projetados dos trabalhadores (por exemplo, tempo de ocupação, carga horária e renda) e do ambiente de trabalho (por exemplo, alunos por turma), de forma a subsidiar políticas públicas direcionadas de prevenção de ausência laboral, evitando novos adoecimentos, melhorando a qualidade de vida desses profissionais e, consequentemente, a qualidade do ensino na rede pública. 


\section{MÉTODOS}

Trata-se de um estudo transversal para predizer risco de afastamento por morbidade (doença ou doença relacionada ao trabalho) em professores que atuam na educação infantil (período pré-escolar) de toda a rede pública municipal do estado de São Paulo, pelo uso de dados secundários, públicos e anônimos.

\section{Fontes de Dados}

Foram utilizados dados individualizados em seu menor nível de desagregação, conhecidos também como microdados ${ }^{11}$, de vínculos empregatícios formais fornecidos pela RAIS no período entre 2014 e 2018. A RAIS mantém informações fornecidas anualmente pelas empresas públicas e privadas sobre os vínculos empregatícios formais de seus funcionários, como contratações e demissões realizadas no ano corrente, além de dados sobre jornada de trabalho e afastamentos por doença ou doença relacionada ao trabalho. Foram incluídos professores da rede pública municipal de todos os municípios do estado de São Paulo que informaram dados à RAIS, nos quais os docentes possuíam nível superior e vínculo empregatício ativo até 31 de dezembro do ano corrente atuando no ensino pré-escolar, classificados na RAIS com a CBO 231105 (Professores de Ensino Superior no Ensino Infantil, quatro a seis anos).

Como forma de identificação do desfecho, foram utilizados os campos de causa de afastamento presentes na RAIS, filtrando-se pelos códigos 30 (doença de trabalho) e 40 (doença). Não foram consideradas aposentadorias decorrentes de doenças. Para a identificação do tipo de estabelecimento (federal, estadual, municipal ou privado), foi utilizada a informação da natureza jurídica do estabelecimento (códigos 1031, 1066, 1120, 1155, 1180 e 1244). Para identificação da atividade do estabelecimento, foram utilizados o código 841160 (administração pública em geral) e a divisão 85 (educação) da Classificação Nacional de Atividades Econômicas versão 2.0 (CNAE 2.0) fornecidos na RAIS.

Cerca de 99,7\% ( $\mathrm{n}=174.346)$ dos registros estavam cadastrados no CNAE 841160, sendo que o restante, $0,03 \%(\mathrm{n}=52)$, pertencia a um CNAE não relacionado ao ensino infantil pré-escolar, sendo excluído da seleção. Também foram excluídos registros em que o município de exercício do trabalhador não correspondia à unidade federativa de São Paulo, resultando no total de 174.294 registros. Nas situações em que o município de exercício do trabalhador não foi informado, foi adotado o município do estabelecimento.

Para capturar parte das condições de trabalho a que esses profissionais estavam expostos, foi vinculada a informação da média de alunos por turma na etapa pré-escolar, fornecida pelo Instituto Nacional de Estudos e Pesquisas Educacionais Anísio Teixeira (Inep) ${ }^{14}$, considerando que algumas doenças comuns aos docentes estão associadas à acústica do ambiente e à complexidade na atuação do docente ${ }^{3}$, e adicionado o número de habitantes por município, fornecido pela Fundação Seade ${ }^{15}$. Os dados do Inep e da Fundação Seade foram vinculados à amostra selecionada da RAIS por meio do código do município no Instituto Brasileiro de Geografia e Estatística (IBGE).

Ao todo, foram selecionadas 11 variáveis preditoras: sexo, idade, pós-graduação, tamanho do estabelecimento, tipo de vínculo empregatício, primeiro emprego, tempo no emprego, horas contratadas, média anual da quantidade de salários mínimos, média de alunos por turma e número de habitantes no município de atuação do trabalhador.

\section{Preparação dos Dados}

Os dados passaram por uma etapa inicial de pré-processamento para tratar as variáveis ausentes e transformá-los para que pudessem ser utilizados na construção dos diferentes tipos de modelos preditivos de machine learning ${ }^{16}$. Todas as variáveis selecionadas estavam totalmente preenchidas, sem valores ausentes (missing). Variáveis com mais de duas categorias foram representadas por um conjunto de variáveis chamadas dummy, em que para cada categoria é gerada uma nova variável com valores de 0 ou 1 . As variáveis contínuas foram 
padronizadas por meio de escore-z (z-score). Foi testada a correlação entre as variáveis numéricas e constatada alta correlação entre o número de habitantes no município e a média de alunos $(0,93)$, portanto optou-se por dicotomizar a variável de número de habitantes utilizando a definição de 500 mil habitantes para identificar atuação em cidade grande ${ }^{17}$.

Para a fase de pré-processamento e carga de dados, foi utilizado o sistema gerenciador de banco de dados MS SQL Server. Para a análise de dados e construção dos modelos preditivos, foi utilizado o software R.

\section{Construção dos Modelos Preditivos}

Ao todo, foram desenvolvidos cinco algoritmos de aprendizado supervisionado de machine learning: regressão logística, árvores de decisã ${ }^{16}$, random forest $^{18}, X$ GBoost $t^{19}$ e redes neurais artificiais ${ }^{20}$. Algoritmos de machine learning estão sujeitos a problemas como o subajuste (underfitting) - quando o modelo não consegue se ajustar à variabilidade inerente dos dados, gerando estimativas ruins - e sobreajuste (overfitting) - quando o modelo se ajusta muito bem para a amostra utilizada no treinamento, mas não obtém bons resultados para novos dados. Uma forma de melhorar o desempenho dos algoritmos e evitar problemas como overfitting ou undefitting é aplicar técnicas de reamostragem (resampling) e validação cruzada (cross-validation) para a seleção de hiperparâmetros ${ }^{16}$.

Na validação cruzada, o conjunto de dados é separado em duas partes. A primeira parte é destinada ao treinamento do algoritmo e a segunda parte é utilizada para ajustes dos hiperparâmetros do modelo, simulando novos dados e selecionando hiperparâmetros que otimizem a métrica de performance escolhida. Essa fase de treinamento permite que o algoritmo obtenha um melhor desempenho preditivo quando são apresentados novos conjuntos de dados. Uma das técnicas de validação cruzada mais conhecidas é a $k$-fold, na qual $k$ significa o número de subconjuntos dos dados de testes que serão utilizados para ajustes dos hiperparâmetros do modelo durante a fase de treinamento ${ }^{21}$.

Para a construção dos modelos, separou-se uma parte dos dados (60\%) referente ao período entre 2014 e 2016 ( $n=103.357$ ) para treinamento, incluindo validação cruzada com a técnica $k$-fold utilizando 10 partições para definição dos hiperparâmetros. A outra parte dos registros (40\%), referente ao período entre 2017 e 2018 ( $\mathrm{n}=70.937)$, foi utilizada para a avaliação de desempenho dos modelos, omitindo-se o resultado sobre afastamento por doença e aplicando o modelo treinado para estimar o desfecho em dados futuros. Para avaliar o desempenho dos modelos, foram analisadas medidas como acurácia, sensibilidade, especificidade, valor preditivo positivo (PPV) e valor preditivo negativo (NPV). Para selecionar o melhor modelo, foi utilizado o valor da área abaixo da curva receiver operating characteristic (AUROC) ${ }^{22}$. Foi utilizado o coeficiente kappa de Cohen para avaliar a concordância entre os valores previstos e observados.

Este estudo segue as orientações de descrição de modelos preditivos multivariados para diagnóstico ou prognóstico (Transparent Reporting of a Multivariable Prediction Model for Individual Prognosis or Diagnosis - TRIPOD) ${ }^{23}$.

\section{Critérios de Confidencialidade}

O projeto foi aprovado pelo Comitê de Ética em Pesquisa da Faculdade de Saúde Pública da Universidade de São Paulo, sob o no 4.031.362, CAAE 30786620.5.0000.5421.

\section{RESULTADOS}

\section{População de Estudo}

Foi realizada inicialmente a análise descritiva dos dados de treinamento (2014 a 2016) e teste (2017 a 2018). Os dados de treinamento compreendem 103.357 registros de vínculos 
Tabela 1. Análise descritiva dos dados de treinamento $(n=103.357)$ e teste $(n=70.937)$.

\begin{tabular}{|c|c|c|c|c|c|}
\hline \multirow{2}{*}{ Variável } & \multirow{2}{*}{ Categoria } & \multicolumn{2}{|c|}{ Treinamento } & \multicolumn{2}{|c|}{ Teste } \\
\hline & & $\mathbf{n}$ & $\%$ & $\mathbf{n}$ & $\%$ \\
\hline \multirow[t]{2}{*}{ Sexo } & 0 - Masculino & 22.120 & 21,40 & 16.055 & 22,63 \\
\hline & 1 - Feminino & 81.237 & 78,60 & 54.882 & 77,37 \\
\hline \multirow[t]{2}{*}{ Pós-graduação } & 0 - Não & 100.904 & 97,63 & 68.412 & 96,44 \\
\hline & $1-\operatorname{Sim}$ & 2.453 & 2,37 & 2.525 & 3,56 \\
\hline Idade $^{a}$ & - & \multicolumn{2}{|c|}{$43,74(9,73)$} & \multicolumn{2}{|c|}{$43,97(9,70)$} \\
\hline \multirow[t]{2}{*}{ Empresa grande $^{b}$} & 0 - Não & 19 & 0,02 & 19 & 0,03 \\
\hline & $1-\operatorname{Sim}$ & 103.338 & 99,98 & 70.918 & 99,97 \\
\hline \multirow[t]{3}{*}{ Tipo de vínculo } & 0 - Estatutário & 96.682 & 93,54 & 65.210 & 91,93 \\
\hline & $1-\mathrm{CLT}$ & 5.867 & 5,68 & 4.493 & 6,33 \\
\hline & 2 - Temporário & 808 & 0,78 & 1.234 & 1,74 \\
\hline \multirow[t]{2}{*}{ Primeiro emprego } & 0 - Não & 100.105 & 96,85 & 65.236 & 91,96 \\
\hline & $1-\operatorname{Sim}$ & 3.252 & 3,15 & 5.701 & 8,04 \\
\hline $\begin{array}{l}\text { Tempo no emprego } \\
\text { (meses) }^{a}\end{array}$ & - & \multicolumn{2}{|c|}{$120,49(92,31)$} & \multicolumn{2}{|c|}{$123,29(93,84)$} \\
\hline Horas contratadas ${ }^{\mathrm{a}}$ & - & \multicolumn{2}{|c|}{$33,28(6,60)$} & \multicolumn{2}{|c|}{$33,28(6,33)$} \\
\hline Salários mínimos ${ }^{\mathrm{a}}$ & - & \multicolumn{2}{|c|}{$5,03(3,04)$} & \multicolumn{2}{|c|}{$5,04(3,04)$} \\
\hline \multirow[t]{2}{*}{ Cidade grande $^{c}$} & 0 - Até 500 mil hab. & 30.866 & 29,86 & 22.212 & 31,31 \\
\hline & 1 - Acima de 500 mil hab. & 72.491 & 70,14 & 48.725 & 68,69 \\
\hline Alunos por turma ${ }^{a}$ & - & \multicolumn{2}{|c|}{$27,42(5,75)$} & \multicolumn{2}{|c|}{$26,74(5,50)$} \\
\hline
\end{tabular}

hab.: habitantes

a Média (desvio-padrão).

b Acima de 100 funcionários.

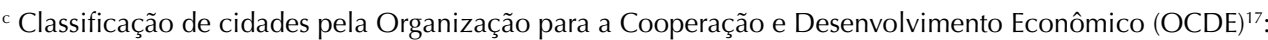
pequena, média, grande (acima de 500 mil habitantes) e metrópole.

empregatícios de professores, havendo 45.419 (43,94\%) registros de afastamentos por doença ou doença relacionada ao trabalho. Os dados de teste correspondem a 70.937 registros e 30.261 casos de afastamento por doença (42,65\%). A Tabela 1 mostra os resultados dessa análise.

Em seguida, foi realizada a análise das variáveis independentes para verificar se há relação significativa com o desfecho, utilizando o teste de associação pelo qui-quadrado de Pearson para as variáveis categóricas. A Tabela 2 mostra os resultados e permite observar que a maioria das variáveis apresenta associação significativa com o desfecho.

\section{Análise dos Modelos Preditivos}

A Figura 1 mostra as curvas ROC de cada modelo desenvolvido, nos quais foram incluídas 11 variáveis (tipo de vínculo empregatício, número de horas contratadas, tempo de emprego em meses, média da quantidade de salários mínimos do ano, sexo, tamanho do estabelecimento, possuir pós-graduação, idade, primeiro emprego, média de alunos por turma pré-escolar no município e número de habitantes). 
Tabela 2. Análise de associação com o desfecho da amostra de treinamento.

\begin{tabular}{|c|c|c|c|c|c|}
\hline \multirow{3}{*}{ Variável } & \multirow{3}{*}{ Categoria } & \multicolumn{3}{|c|}{ Professores afastados por doença } & \multirow{3}{*}{ Teste $c^{2}$} \\
\hline & & Sim & Não & Total & \\
\hline & & n (\%) & n (\%) & n (\%) & \\
\hline \multicolumn{6}{|l|}{ Dados do trabalhador } \\
\hline \multirow[t]{2}{*}{ Sexo } & 0 - Masculino & $9.930(44,89)$ & $12.190(55,11)$ & $22.120(100,0)$ & $<0,001$ \\
\hline & 1 - Feminino & $35.489(43,69)$ & $45.748(56,31)$ & $81.237(100,0)$ & \\
\hline \multirow[t]{2}{*}{ Pós-graduação } & 0 - Não & $45.075(44,67)$ & $55.829(55,33)$ & $100.904(100,0)$ & \multirow{2}{*}{$<0,0001$} \\
\hline & $1-\operatorname{Sim}$ & $344(14,02)$ & $2.109(85,98)$ & $2.453(100,0)$ & \\
\hline Idade & $43,74(9,73)$ & & & & \\
\hline \multicolumn{6}{|l|}{ Dados da empresa } \\
\hline \multirow[t]{2}{*}{ Empresa grande $^{b}$} & 0 - Não & $11(57,89)$ & $8(42,11)$ & $19(100,00)$ & 0,220 \\
\hline & $1-\operatorname{Sim}$ & $45.408(43,94)$ & $57.930(56,06)$ & $103.338(100,00)$ & \\
\hline \multicolumn{6}{|l|}{ Dados do vínculo } \\
\hline \multirow[t]{3}{*}{ Tipo de vínculo } & $0-\mathrm{CLT}$ & $746(12,72)$ & $5.121(87,28)$ & $5.867(100,0)$ & $<0,0001$ \\
\hline & 1 - Estatutário & $44,500(46,03)$ & $52.182(53,97)$ & $96.682(100,0)$ & \\
\hline & 2 - Temporário & $173(21,41)$ & $635(78,59)$ & $808(100,0)$ & \\
\hline \multirow[t]{2}{*}{ Primeiro emprego } & 0 - Não & $44.616(44,57)$ & $55.489(55,43)$ & $100.105(100,0)$ & $<0,0001$ \\
\hline & $1-\operatorname{Sim}$ & $803(24,69)$ & $2.449(75,31)$ & $3.252(100,0)$ & \\
\hline $\begin{array}{l}\text { Tempo no emprego } \\
\text { (meses) }\end{array}$ & $120,49(92,31)$ & & & & \\
\hline Horas contratadas & $33,28(6,60)$ & & & & \\
\hline Salários mínimos & $5,03(3,04)$ & & & & \\
\hline \multicolumn{6}{|l|}{ Dados ambientais } \\
\hline \multirow[t]{2}{*}{ Cidade grande $^{c}$} & $\begin{array}{l}0-\text { Até } \\
500 \text { mil hab. }\end{array}$ & $3.422(11,09)$ & $27.444(88,91)$ & $30.866(100,0)$ & $<0,0001$ \\
\hline & $\begin{array}{l}1-\text { Acima de } \\
500 \text { mil hab. }\end{array}$ & $41.997(57,93)$ & $30.494(42,07)$ & $72.491(100,0)$ & \\
\hline Alunos por turma & $27,04(5,60)$ & & & & \\
\hline
\end{tabular}

hab.: habitantes

a Teste de associação pelo $c^{2}: H_{0}$ : não existe associação; $\mathrm{H}_{\mathrm{a}}$ : existe associação.

${ }^{\mathrm{b}}$ Acima de 100 funcionários.

${ }^{\text {c }}$ Classificação de cidades pela Organização para a Cooperação e Desenvolvimento Econômico (OCDE) ${ }^{17}$ : pequena, média, grande (acima de 500 mil habitantes) e metrópole.

Nota: Valores em média (desvio-padrão).

A Tabela 3 mostra os resultados do desempenho de cada algoritmo. O melhor modelo foi o de redes neurais artificiais, que obteve 0,79 de área abaixo da curva, com acurácia de $71,52 \%$, sensibilidade de $72,86 \%$, especificidade de $70,52 \%$, PPV de $64,77 \%$ e NPV de $77,74 \%$. O modelo também apresentou a melhor coeficiente kappa, de 0,4266. 


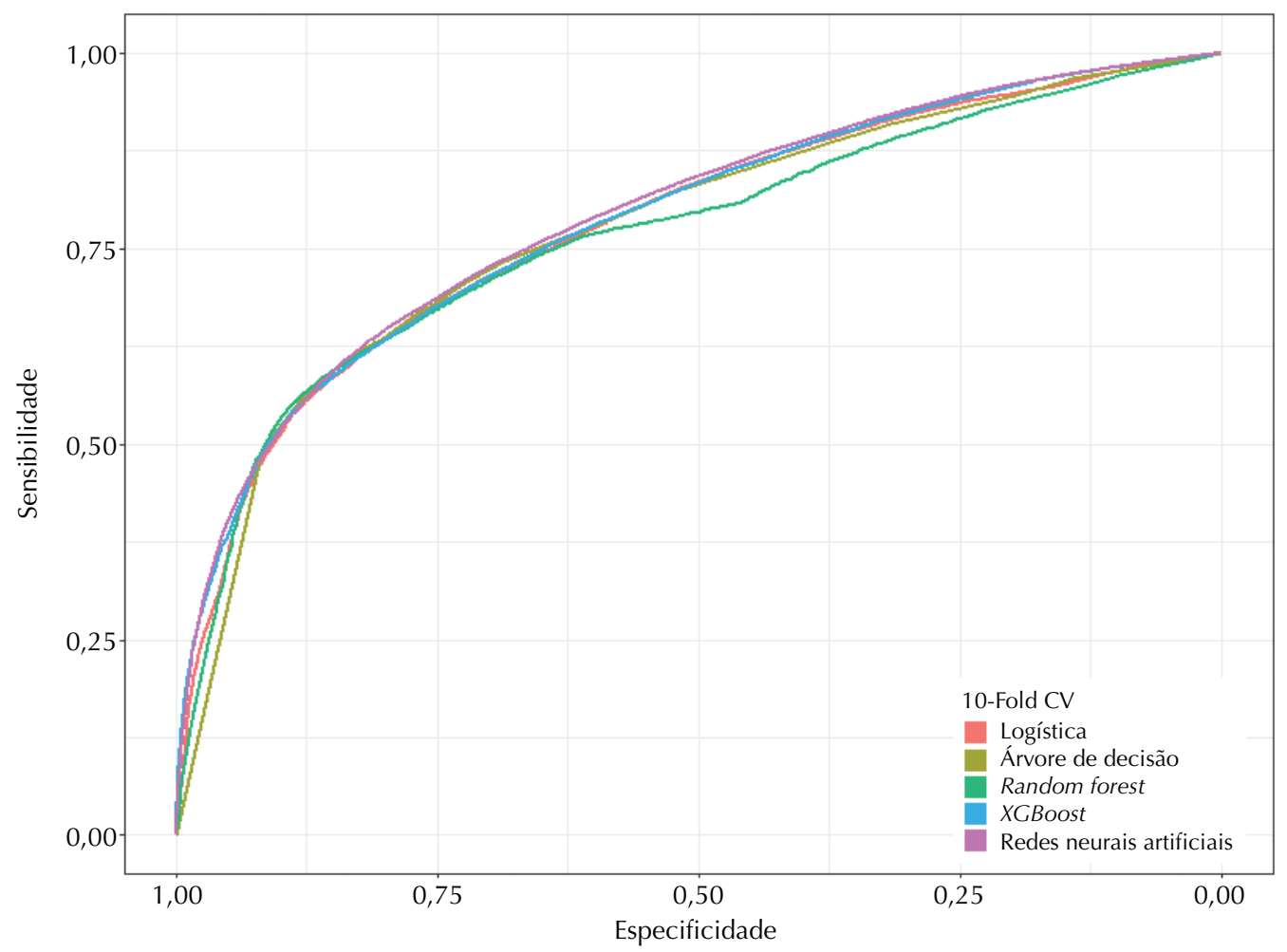

Figura 1. Comparação dos modelos com base nos resultados de predição utilizando dados de teste (2017 e 2018).

Tabela 3. Resultados dos modelos desenvolvidos utilizando dados de teste.

\begin{tabular}{lccccccc}
\hline Algoritmo & AUC & Sensibilidade & Especificidade & Acurácia & PPV & NPV & Kappa \\
\hline $\begin{array}{l}\text { Regressão } \\
\text { logística }\end{array}$ & $\begin{array}{c}0,7792 \\
{[0,7759-0,7826]}\end{array}$ & 0,7754 & 0,6568 & 0,7074 & 0,6270 & 0,7972 & 0,4195 \\
$\begin{array}{l}\text { Árvore de } \\
\text { decisão }\end{array}$ & $\begin{array}{c}0,7756 \\
{[0,7722-0,7790]}\end{array}$ & 0,7008 & 0,7243 & 0,7142 & 0,6541 & 0,7649 & 0,4212 \\
Random forest & $\begin{array}{c}0,7670 \\
{[0,7634-0,7703]}\end{array}$ & 0,7715 & 0,6571 & 0,7059 & 0,6260 & 0,7944 & 0,4134 \\
XGBoost & $\begin{array}{c}0,7843 \\
{[0,7810-0,7876]}\end{array}$ & 0,7235 & 0,6970 & 0,7083 & 0,6398 & 0,6970 & 0,4136 \\
$\begin{array}{l}\text { Redes neurais } \\
\text { artificiais }\end{array}$ & $\begin{array}{c}0,7902 \\
{[0,7867-0,7934]}\end{array}$ & 0,7286 & 0,7052 & 0,7152 & 0,6477 & 0,7774 & 0,4266 \\
\hline AUC: án
\end{tabular}

AUC: área abaixo da curva

Os hiperparâmetros dos modelos de machine learning que obtiveram os maiores valores de AUC foram os seguintes: árvore de decisão (cp-complexity-parameter $=0.000385$ ), random forest $(\mathrm{mtry}=7)$, XGBoost (nrounds $=100$, max_depth $=4$, gamma $=0.6$, colsample_bytree $=0.8$, min_child_weight $=2$, subsample $=1$ ) e redes neurais artificiais $($ size $=19$, decay $=0.2)$.

Cada algoritmo utiliza um conjunto de regras diferentes para se ajustar aos dados com o menor erro possível, sendo que algumas variáveis se tornam mais importantes que outras para a capacidade preditiva do algoritmo. A importância das variáveis refere-se a quanto a variável contribuiu para realizar predições acuradas em cada modelo. Para os modelos lineares, utiliza-se de estatística t tradicional para contabilizar a importância das variáveis. Nos demais modelos, a importância depende da implementação de cada algoritmo.

Por exemplo, no caso de árvores de decisão, se uma variável é utilizada com maior frequência nas divisões de folhas contribuindo para maior pureza do nó, ela terá uma importância maior para o modelo final, significando que sua remoção prejudicará a performance geral do 

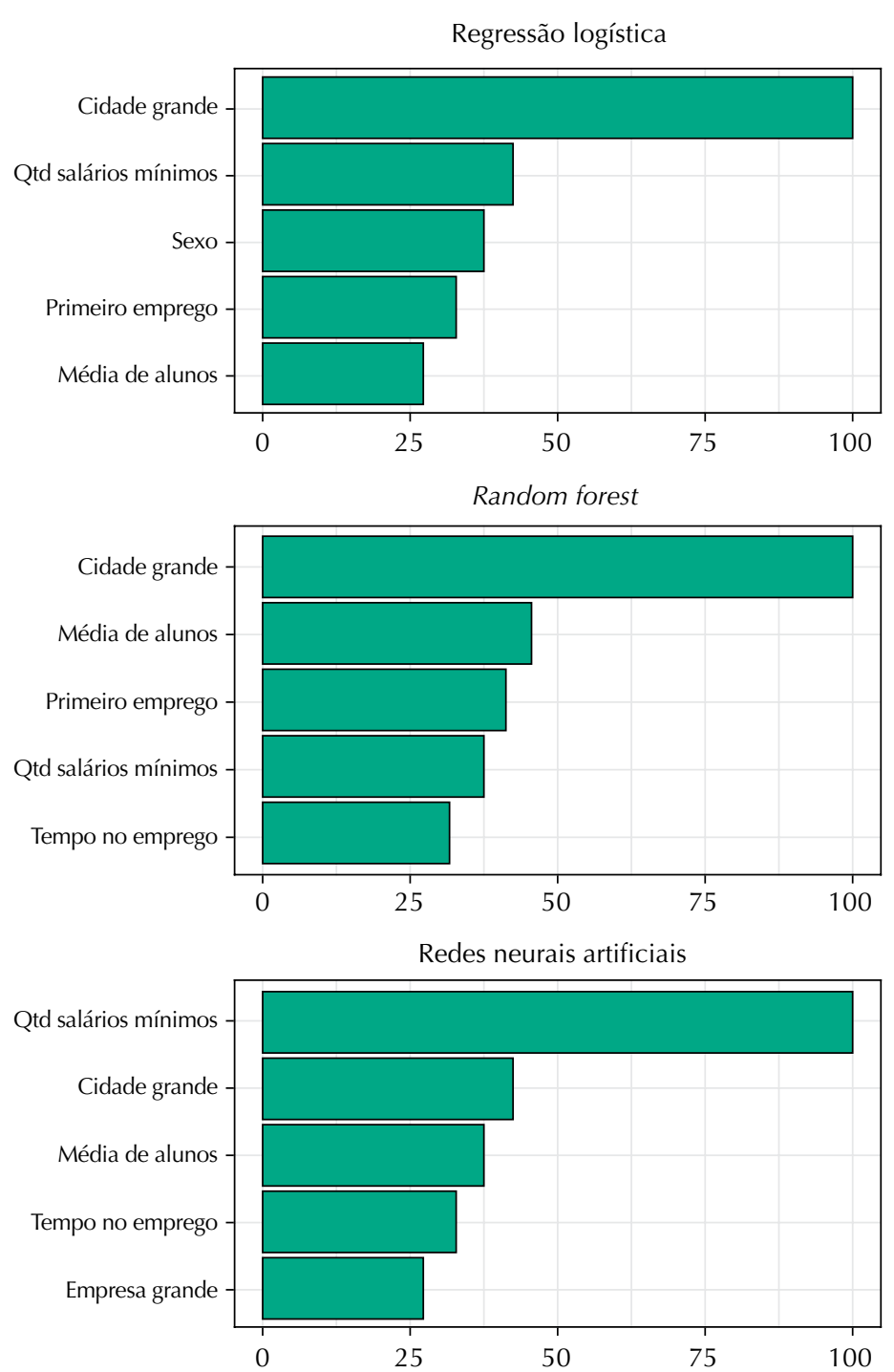
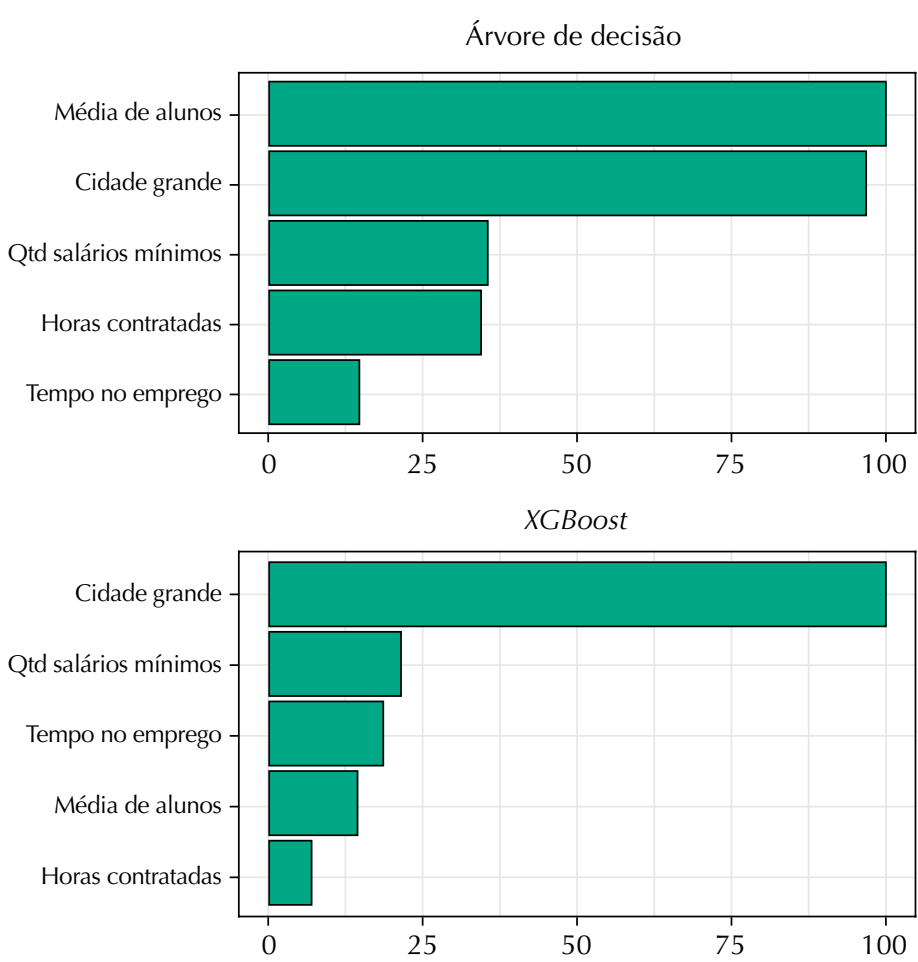

Figura 2. Importância das variáveis por modelo preditivo.

modelo. Nos modelos que utilizam múltiplas árvores, como o random forest, a importância das variáveis é calculada a cada divisão das folhas de uma árvore e somada com o resultado das demais árvores ${ }^{21}$. Nos modelos de redes neurais, para este estudo foi utilizado o método "weights"24, em que se utiliza uma combinação dos valores de saída dos neurônios das camadas ocultas e os valores dos pesos de cada conexão para calcular a maior contribuição e representatividade de cada variável de entrada no resultado da classificação. Neste estudo, foi utilizado o pacote $\operatorname{caret}^{25}$ do R, que já implementa o cálculo de importância de variáveis de acordo com cada modelo ${ }^{26}$.

Apesar de não ter interpretação causal ${ }^{27}$, as variáveis podem dar informações relevantes sobre a sua relação com o desfecho. A Figura 2 demonstra como cada variável contribuiu para os modelos desenvolvidos.

As variáveis que mais contribuíram para o melhor modelo (redes neurais artificiais) foram: quantidade de salários mínimos, cidade grande, média de alunos por turma, tempo no emprego e empresa grande.

\section{DISCUSSÃO}

A RAIS pode fornecer indícios da situação de morbidade dos trabalhadores por meio do campo de causa de afastamento. A ampla cobertura e o registro individualizado facilitam 
a seleção e análise de dados de uma população de trabalhadores formais com a mesma ocupação em um determinado estado, o que permite acompanhar ao longo dos anos a evolução de afastamentos por doença e predizer quais profissionais apresentam maior risco de adoecimento nos próximos anos. Para o melhor algoritmo (redes neurais artificiais), obteve-se 0,79 da área abaixo da curva ROC, sensibilidade de 72,86\% e 71,52\% de acurácia, usando apenas 11 variáveis preditoras.

Os resultados mostram a viabilidade da utilização de fontes anonimizadas para a predição de morbidade de docentes do ensino pré-escolar com algoritmos de machine learning. As variáveis que mais contribuíram para o melhor modelo podem ser exploradas em estudos futuros, para verificar se há relação causal. Porte da cidade em que se trabalha, renda média do trabalhador e média de alunos por turma podem ter impactos diretos na saúde dos professores. No caso da média de alunos, há evidências na literatura que corroboram este raciocínio, como problemas na acústica do ambiente e elevado ruído devido a maior agitação em sala de aula ${ }^{3,28}$.

Algoritmos de machine learning têm sido utilizados em diferentes contextos na saúde do trabalhador para auxiliar na predição de morbidades relacionadas às atividades destes profissionais ${ }^{29,30}$. Na área da educação, não foram encontrados estudos que utilizaram machine learning ou demais modelos preditivos para risco de absenteísmo docente como principal desfecho, havendo alguns estudos que analisaram a associação de variáveis preditoras com o desfecho ${ }^{31,32}$, não sendo sua predição o principal objetivo. A predição de absenteísmo docente pode identificar professores que precisam de maior atenção, permitindo adotar medidas para evitar afastamentos por doenças relacionadas à ocupação $0^{3,28}$, diminuindo a ausência ao trabalho e consequentemente aprimorando a qualidade do ensino ${ }^{33}$.

O estudo possui algumas limitações. Por se tratar de dados anônimos, não foi possível verificar se os docentes, que podem ter múltiplos vínculos empregatícios municipais, atuam em mais de uma escola. Também não foi possível identificar se houve um afastamento prévio por doença pré-existente que possa estar relacionado aos afastamentos seguintes. Por fim, foi analisado um número baixo de variáveis preditoras devido à disponibilidade da RAIS. Espera-se que no futuro, com uma coleta mais extensa realizada por meio de questionários, sejam possíveis resultados ainda mais robustos por parte dos algoritmos.

Em conclusão, somente com dados públicos e anônimos, foi possível obter boa sensibilidade (73\%) e especificidade (71\%) na identificação de risco de absenteísmo por doença, independentemente da causa da morbidade. Apesar de não ter sido criada para fins da saúde, a RAIS mostrou-se viável para análise de absenteísmo por morbidades, principalmente por conter variáveis das relações de trabalho, podendo ser explorada em outras categorias profissionais. Os modelos desenvolvidos podem futuramente ser aplicados nas demais etapas de ensino e em outras categorias ocupacionais. Algoritmos de machine learning podem contribuir com predições mais assertivas na análise de absenteísmo de professores do ensino infantil da rede pública municipal, podendo ser usados na elaboração de indicadores de morbidade ou para subsidiar políticas públicas prevencionistas direcionadas a essa categoria profissional.

\section{REFERÊNCIAS}

1. DIEESE. Anuário do Sistema Público de Emprego, Trabalho e Renda: mercado de trabalho 2016. São Paulo; 2016.

2. Gasparini SM, Barreto SM, Assunção AA. O professor, as condições de trabalho e os efeitos sobre sua saúde. Educ Pesqui. 2005;31(2):189-99. https://doi.org/10.1590/S1517-97022005000200003

3. Medeiros AM, Vieira MT. Ausência ao trabalho por distúrbio vocal de professores da Educação Básica no Brasil. Cad Saude Publica. 2019;35 Supl 1:e00171717. https://doi.org/10.1590/0102-311x00171717 
4. Arcoverde, L, Franco E, Galvão D, Prado G. Número de professores afastados por transtornos em SP quase dobra em 2016 e vai a 50 mil. G1 Globo News (São Paulo Ed.). 21 nov 2017 [citado 7 fev 2020]. Disponível em: https://g1.globo.com/sp/sao-paulo/noticia/numero-deprofessores-afastados-por-transtornos-em-sp-quase-dobra-em-2016-e-vai-a-50-mil.ghtml

5. Rodríguez-Loureiro L, Artazcoz L, López-Ruiz M, Assunção AA, Benavides FG. Joint effect of paid working hours and multiple job holding on work absence due to health problems among basic education teachers in Brazil: the Educatel Study. Cad Saude Publica. 2019;35 Supl 1:e00081118. https://doi.org/10.1590/0102-311x00081118

6. Silva J, Fischer FM. Invasão multiforme da vida pelo trabalho entre professores de educação básica e repercussões sobre a saúde. Rev Saude Publica. 2020;54:03. https://doi.org/10.11606/s1518-8787.2020054001547

7. Assunção AA, Oliveira DA. Intensificação do trabalho e saúde dos professores. Educ Soc. 2009;30(107):349-72. https://doi.org/10.1590/S0101-73302009000200003

8. Porto LA, Oliveira NF, Carvalho FM, Araújo TM. Construção de um índice de morbidade para professoras da educação básica. Rev Baiana Saude Publica. 2008;32(2):282-96. https://doi.org/10.22278/2318-2660.2008.v32.n2.a1449

9. Maia EG, Claro RM, Assunção AA. Múltiplas exposições ao risco de faltar ao trabalho nas escolas da Educação Básica no Brasil. Cad Saude Publica. 2019;35 Supl 1:e00166517. https://doi.org/10.1590/0102-311x00166517

10. Portal Brasileiro de Dados Abertos. Primeira Lei de Acesso no mundo que prevê dados abertos. Brasília, DF; 2018 [citado 1 out 2018]. Disponível em: http://dados.gov.br/noticia/primeira-leide-acesso-no-mundo-que-preve-dados-abertos

11. Ministério do Trabalho (BR). PDET - Progama de Disseminação das Estatísticas do Trabalho. Microdados RAIS e CAGED. Brasília, DF; 2019 [citado 7 fev 2020]. Disponível em: http://pdet.mte.gov.br/microdados-rais-e-caged

12. Brasil. Decreto No 76.900, de 23 de dezembro de 1975. Institui a Relação Anual de Informações Sociais - RAIS e dá outras providências. Brasília, DF; 1975 [citado 28 fev 2020]. Disponível em: http://www.planalto.gov.br/ccivil_03/decreto/Antigos/D76900.htm

13. Fernandes FT, Chiavegatto Filho ADP. Perspectivas do uso de mineração de dados e aprendizado de máquina em saúde e segurança no trabalho. Rev Bras Saude Ocup. 2019;44:e13. https://doi.org/10.1590/2317-6369000019418

14. Instituto Nacional de Estudos e Pesquisas Educacionais Anísio Teixeira. Indicadores Educacionais. Brasília, DF: INEP; 2019 [citado 18 set 2019]. Disponível em: http://portal.inep.gov.br/web/guest/indicadores-educacionais

15. Fundação Sistema Estadual de Análise de Dados. Informações dos Municípios Paulistas - IMP. São Paulo: SEADE; 2019 [citado 18 fev 2019]. Disponível em: http://www.imp.seade.gov.br/frontend/\#/

16. Kuhn M, Johnson K. Applied predictive modeling. New York: Springer Science \& Business Media; 2013

17. OECD Data. Urban population by city size. Paris; 2018 [citado 18 fev 2019]. Disponível em: https://data.oecd.org/popregion/urban-population-by-city-size.htm

18. Breiman L. Random forests. Mach Learn. 2001;45:5-32. https://doi.org/10.1023/A:1010933404324

19. Chen T, Guestrin C. XGBoost: a scalable tree boosting system. In: Proceedings of the 22nd ACM SIGKDD International Conference on Knowledge Discovery and Data Mining; 2016 Aug; San Francisco, CA. New York: Association for Computing Machinery; 2016. p.785-94.

20. Bishop C. Neural networks for pattern recognition. Oxford (UK): Oxford University Press; 1995.

21. Hastie T, Tibshirani R, Friedman J. The elements of statistical learning. 2. ed. New York: Springer Science \& Business Media; 2016.

22. Bradley AP. The use of the area under the ROC curve in the evaluation of machine learning algorithms. Pattern Recognit. 1997;30(7):1145-59. https://doi.org/10.1016/S0031-3203(96)00142-2

23. Moons KGM, Altman DG, Reitsma JB, loannidis JPA, Macaskill P, Steyerberg EW, et al. Transparent reporting of a multivariable prediction model for individual prognosis or diagnosis (TRIPOD): explanation and elaboration. Ann Intern Med. 2015;162(1):W1-73. https://doi.org/10.7326/M14-0698 
24. Gevrey M, Dimopoulos I, Lek S. Review and comparison of methods to study the contribution of variables in artificial neural network models. Ecol Modell. 2003;160(3):249-64. https://doi.org/10.1016/S0304-3800(02)00257-0

25. Kuhn M, Wing J, Weston S, Williams A, Keefer C, Engelhardt A, et al. A Short Introduction to the caret package. 2020 [citado 22 set 2020]. Disponível em: https://cran.r-project.org/web/packages/caret/vignettes/caret.html

26. Kuhn M. Variable importance. 2019 [citado 11 ago 2020]. Disponível em: https://topepo.github.io/caret/variable-importance.html

27. Santos HG. Comparação da performance de algoritmos de machine learning para a análise preditiva em saúde pública e medicina [tese]. São Paulo: Faculdade de Saúde Pública da Universidade de São Paulo; 2018.

28. Rezende BA, Medeiros AM, Silva AM, Assunção AA. Fatores associados à percepção de ruído ocupacional intenso pelos professores da educação básica no Brasil. Rev Bras Epidemiol. 2019;22:e190063. https://doi.org/10.1590/1980-549720190063

29. Aliabadi M, Farhadian M, Darvishi E. Prediction of hearing loss among the noise-exposed workers in a steel factory using artificial intelligence approach. Int Arch Occup Environ Health. 2015;88(6):779-87. https://doi.org/10.1007/s00420-014-1004-z

30. Lee YC, Huang SC, Huang $\mathrm{CH}, \mathrm{Wu} \mathrm{HH}$. A new approach to identify high burnout medical staffs by kernel K-means cluster analysis in a regional teaching hospital in Taiwan. Inquiry. 2016;53:0046958016679306. https://doi.org/10.1177/0046958016679306

31. Ferris G, Bergin TG, Wayne SJ. Personal characteristics, job performance, and absenteeism of public school teachers. J Appl Soc Psychol. 1988;18(7):552-63. https://doi.org/10.1111/j.1559-1816.1988.tb00036.x

32. Rosenblatt Z, Shirom A. Predicting teacher absenteeism by personal background factors. J Educ Adm. 2005;43(2):209-25. https://doi.org/10.1108/09578230510586597

33. Miller RT, Murnane RJ, Willett JB. Do teacher absences impact student achievement? Longitudinal evidence from one urban school district. Educ Eval Policy Anal. 2008;30(2):181-200. https://doi.org/10.3102/0162373708318019

Contribuição dos Autores: Concepção e planejamento do estudo: FTF, ADPCF. Coleta dos dados: FTF. Treinamento dos modelos: FTF. Análise e interpretação dos resultados: FTF, ADPCF. Elaboração e revisão do manuscrito: FTF, ADPCF. Aprovação da versão final: FTF, ADPCF. Responsabilidade pública pelo conteúdo do artigo: FTF.

Conflito de Interesses: Os autores declaram não haver conflito de interesses. 